\section{Nível de atividade física e fatores associados em adultos no Município de Joaçaba, Santa Catarina, Brasil}

\author{
Physical activity and associated factors among \\ adults in Joaçaba, Santa Catarina, Brazil
}

\section{${ }_{1}$ Programa de \\ Pós-graduação em Saúde Coletiva, Universidade do Oeste de Santa Catarina, Joaçaba, Brasil. \\ 2 Programa de Pós- graduação em Saúde Pública, Universidade Federal \\ de Santa Catarina, \\ Florinópolis, Brasil \\ Correspondência K. G. Peres \\ Programa de Pós- \\ graduação em Saúde Pública Universidade Federal de Santa Catarina, \\ Campos Universitário Trindade, Florianópolis, SC 88010-970, Brasil. \\ karengp@ccs.ufsc.b}

\begin{abstract}
We investigated the prevalence of physical inactivity and associated factors among the adult population in Joaçaba, Santa Catarina, Brazil, using a cross-sectional, population-based model with subjects from 20 to 59 years of age $(n=579)$. The short version of the International Physical Activity Questionnaire (IPAQ) was used to define the level of physical activity. Descriptive crude analyses and Poisson regression were performed to test the associated variables. The response rate was 99.6\%, and prevalence of physical inactivity was 57.4\% (95\%CI: 53.4-61.4). Physical inactivity was associated with not working or not being retired, income (> U\$500/month), age > 30, body mass index above or below standard, and self-reported difficulty with mobility, regardless of gender. The physical inactivity level was lower than in other population-based Brazilian studies. The results may help promote regular physical activity through appropriate public policies.
\end{abstract}

Motor Activity; Adult; Prevalence
Elisabeth Baretta 1

Marly Baretta 1

Karen Glazer Peres 2

\section{Introdução}

A atividade física tem sido considerada um meio de preservar e melhorar a saúde 1 . Sedentarismo e estilos de vida que incorporam pouca atividade física têm sido observados, gerando preocupação por parte dos órgãos de saúde pública no Brasil 2.

A prática de atividade física tem se mostrado benéfica na redução de diversos fatores de risco, propiciando, por exemplo, melhora no metabolismo das gorduras e carboidratos, controle de peso corporal e, muitas vezes, controle da hipertensão ${ }^{3}$. Essa prática contribui também para a manutenção de ossos, músculos e articulações mais saudáveis; diminui os sintomas de depressão e ansiedade, estando, ainda, associada à prevenção de enfermidades como diabetes mellitus, doenças cardiovasculares, osteoporose e alguns tipos de câncer, como os de cólon e mama 2.

Dessa forma, a atividade física, além de poder contribuir para a melhoria da qualidade de vida dos indivíduos, gera economia de recursos financeiros com tratamentos médicos ${ }^{4}$.

No Brasil, a inatividade física, considerada nos momentos de lazer, é mais prevalente entre mulheres, idosos e indivíduos de baixo nível sócio-econômico 2,5 , porém pesquisas de base populacional regionais ainda são escassas em nosso país 6 .

Os objetivos deste estudo foram estimar o nível de atividade física praticado pela população 
adulta do Município de Joaçaba, Santa Catarina, Brasil, e conhecer os fatores associados nesta população.

\section{Método}

Esta pesquisa foi realizada em Joaçaba, município do meio oeste do Estado de Santa Catarina, que possui, aproximadamente, 24.030 habitantes 7 . Realizou-se um estudo transversal, de base populacional, com indivíduos na faixa etária entre 20 e 59 anos de idade, de ambos os sexos, residentes na zona urbana. Obteve-se uma amostra aleatória, em duplo estágio, tendo como primeiro estágio os setores censitários e, como unidades do segundo estágio, os domicílios. Consideraram-se para o cálculo amostral todos os setores censitários urbanos $(\mathrm{n}=22)$. Os parâmetros para o cálculo da amostra foram: tamanho da população igual a 13.307 , prevalência de inatividade física igual a $45 \%$, nível de confiança de $5 \%$ e erro amostral igual a 5 pontos percentuais. O tamanho da amostra foi igual 370 , adicionada de uma correção de 1,4 por causa do desenho do estudo em dois estágios e mais $12 \%$ em virtude de eventuais perdas e/ou recusas, totalizando 580 indivíduos. As residências foram sorteadas com base na divisão dos setores censitários em quarteirões com subseqüente sorteio aleatório do quarteirão e da esquina para início do estudo. Foram coletadas as seguintes informações: sexo; idade em anos completos; peso (kg) e estatura (metros) para o cálculo do índice de massa corporal - IMC $(\leq 18,4 ; 18,5-24,9 ; 25,0-29,9$; $\geq 30,0$ ); estado civil (casado, nunca se casou/mas tem companheiro, nunca se casou/mas não tem companheiro, separado, viúvo, outros); somatório da renda mensal familiar em salários mínimos; escolaridade (número de anos de estudo); situação ocupacional (trabalha, só aposentado, trabalha apesar de aposentado, só dona-de-casa, pensionista, não trabalha, outros) e referente à qualidade de vida (dor impedindo a atividade física - sim/não; capacidade de locomoção auto-relatada - sim/não; energia suficiente para o dia-a-dia - sim/não; satisfação com relação à saúde - sim/não).

A aferição da estatura e da massa corporal foi realizada por meio de uma régua com fita métrica em centímetros e precisão de $1 \mathrm{~mm}$, além de uma balança de plataforma da marca Plenna, com leitura de $0,1 \mathrm{~kg}$. As medidas foram obtidas observando-se os procedimentos recomendados pelo Anthropometric Standardization Reference Manual 8. O IMC foi calculado com base na relação do peso com a altura ao quadrado. Para avaliar o nível de atividade física, utilizou-se o
Questionário Internacional de Atividade Física (IPAQ), versão 8, na sua forma curta ${ }^{9}$. O trabalho de campo foi realizado por duas entrevistadoras e dois auxiliares. A equipe foi submetida a um treinamento para aplicação do questionário e padronização das entrevistas.

O instrumento foi devidamente pré-testado na população adulta de Herval do Oeste, município vizinho. O nível de atividade física foi classificado segundo proposta do IPAQ 10, a saber: insuficientemente ativo, suficientemente ativo e muito ativo.

Os resultados foram analisados no programa estatístico Stata (Stata Corp., College Station, Estados Unidos), versão 9.0. Para analisar as variáveis associadas à inatividade física controlando para potenciais fatores de confusão, foi utilizada a regressão de Poisson. Esta técnica se justifica uma vez que o desfecho estudado pode apresentar uma alta prevalência ( $20 \%$ ou mais), o que provocaria uma superestimativa da magnitude da medida de efeito, odds ratio (OR), obtida por meio da análise de regressão logística 11. Calcularam-se as razões de prevalências (RP) brutas e ajustadas, bem como seus respectivos intervalos de confiança de 95\% (IC95\%). A variável dependente, nível de atividade física, foi dicotomizada em: indivíduos ativos (categorias suficientemente ativo e muito ativo do IPAQ) e indivíduos inativos (categoria insuficientemente ativo do IPAQ). As variáveis idade ( $<29$ anos e 30-59 anos) e anos de estudo (8 anos ou mais; 5-7 anos e menos de 5 anos) foram recategorizadas para a entrada no modelo final.

As variáveis independentes foram inseridas no modelo de regressão de Poisson de maneira crescente, conforme sua significância estatística ( $p<0,20$ ), permanecendo no modelo caso continuassem significantes $(\mathrm{p}<0,05)$ e/ou ajustassem o modelo. A variável sexo foi utilizada para controle.

A pesquisa foi aprovada pelo Comitê de Ética em Pesquisa e os participantes assinaram um termo de consentimento informado.

\section{Resultados}

Foram visitados 215 domicílios e 575 pessoas foram entrevistadas (99,1\%), havendo quatro recusas. Do total dos participantes, $260(45,2 \%)$ eram homens e metade da amostra $(50,4 \%)$ encontrava-se na faixa etária entre 20 e 39 anos. A prevalência de inatividade física foi de 57,4\% (IC95\%: 53,4-61,4) (Tabela 1).

Escore zero de atividade física/semana foi encontrado em $8,8 \%$ nos homens e $5,1 \%$ nas mulheres. A média de minutos/semana de atividade 
física foi de 799,9 (DP = 1076,6) para os homens e 560,7 (DP $=765,5)$ para as mulheres $(\mathrm{p}=0,364)$ (Tabela 2).

Inatividade física apresentou-se associada positivamente aos indivíduos com oito anos ou mais de estudo, àqueles que não trabalhavam e/ou não eram aposentados, a indivíduos com renda superior a mil reais mensais. Observouse uma proporção maior de indivíduos inativos entre aqueles com IMC maior ou menor do que a faixa entre $18,5 \mathrm{~kg} / \mathrm{m}^{2} \mathrm{e} 29,9 \mathrm{~kg} / \mathrm{m}^{2}$, aqueles que auto-avaliaram sentirem pouca ou muita dificuldade de locomoção, indivíduos que não sentiam energia suficiente para o seu dia-a-dia e aqueles que referiram não se sentir bem em relação a sua saúde (Tabela 3).

Através da regressão de Poisson, verificaramse as seguintes variáveis associadas à inatividade física: apresentar idade superior a 29 anos, não estar trabalhando ou não ser aposentado, ter renda mensal superior a mil reais, ter IMC maior do que $29,9 \mathrm{~kg} / \mathrm{m}^{2}$ ou menor do que $18,5 \mathrm{~kg} / \mathrm{m}^{2} \mathrm{e}$ relatar dificuldade de locomoção, independentemente do gênero e das outras variáveis do modelo final (Tabela 4).

Tabela 1

Descrição da amostra, segundo aspectos demográficos e nível de atividade física. Joaçaba, Santa Catarina, Brasil, 2003.

\begin{tabular}{lcc}
\hline Características & $\mathbf{n}$ & $\%$ \\
\hline Sexo & & \% \\
Masculino & 260 & 54,2 \\
Feminino & 315 & 54,8 \\
Faixa etária (anos) & & 25,9 \\
$20-29$ & 149 & 24,5 \\
$30-39$ & 141 & 28,7 \\
$40-49$ & 165 & 20,9 \\
$50-59$ & 120 & 37,7 \\
Atividade física & & 4,9 \\
Insuficientemente ativo & 330 & 100,0 \\
Suficientemente ativo & 217 & 57,4 \\
Muito ativo & 28 & 575 \\
Total & & \\
\hline
\end{tabular}

Tabela 2

Descrição do padrão de atividade física na amostra $(N=575)$. Joaçaba, Santa Catarina, Brasil, 2003.

\begin{tabular}{lccc}
\hline Parâmetros & Amostra & Homens & Mulheres \\
\hline Média (minutos/semana) & 668,9 & $799,9 *$ & 560,7 * \\
Desvio-padrão (minutos/semana) & 926,1 & $1.076,6$ & 765,5 \\
Percentis (minutos/semana) & & 120,0 & 135,0 \\
25 & 125,0 & 342,5 & 300,0 \\
50 & 300,0 & $1.072,5$ & 600,0 \\
75 & 825,0 & 8,8 & 5,1 \\
Porcentagem com escore = 0 (minutos/semana) & 6,8 & 0 & 0 \\
Mínimo (minutos/semana) & 0 & 5.760 & 6.900 \\
Máximo (minutos/semana) & 6.900 & & \\
\hline
\end{tabular}

* Diferença entre sexo masculino e feminino não significativa estatisticamente ( $p=0,364$; Mann-Whitney test). 
Associação entre nível de atividade física, variáveis sócio-demográficas e percepção de qualidade de vida. Joaçaba, Santa Catarina, Brasil, 2003.

\begin{tabular}{|c|c|c|c|c|c|c|c|}
\hline \multirow[t]{3}{*}{ Variável } & \multicolumn{4}{|c|}{ Atividade física } & \multicolumn{2}{|c|}{ Total } & \multirow[t]{3}{*}{$p$ * } \\
\hline & \multicolumn{2}{|c|}{ Ativo } & \multicolumn{2}{|c|}{ Inativo } & & & \\
\hline & $\mathrm{n}$ & $\%$ & $\mathrm{n}$ & $\%$ & $\mathrm{n}$ & $\%$ & \\
\hline \multicolumn{8}{|l|}{ Sexo } \\
\hline Masculino & 115 & 44,2 & 145 & 55,8 & 260 & 100,0 & 0,475 \\
\hline Feminino & 130 & 41,3 & 185 & 58,7 & 315 & 100,0 & \\
\hline \multicolumn{8}{|l|}{ Idade (anos) } \\
\hline $20-29$ & 75 & 50,3 & 74 & 49,7 & 149 & 100,0 & 0,161 \\
\hline $30-39$ & 56 & 39,7 & 85 & 60,3 & 141 & 100,0 & \\
\hline $40-49$ & 68 & 41,2 & 97 & 58,8 & 165 & 100,0 & \\
\hline $50-59$ & 46 & 38,3 & 74 & 61,7 & 120 & 100,0 & \\
\hline \multicolumn{8}{|l|}{ Escolaridade (anos) } \\
\hline 12 & 54 & 32,7 & 111 & 67,3 & 165 & 100,0 & 0,008 \\
\hline $8-11$ & 108 & 45,2 & 131 & 54,8 & 239 & 100,0 & \\
\hline$\leq 7$ & 83 & 48,5 & 88 & 51,5 & 171 & 100,0 & \\
\hline \multicolumn{8}{|l|}{ Ocupação } \\
\hline Trabalha e/ou aposentado & 190 & 45,7 & 226 & 54,3 & 416 & 100,0 & 0,016 \\
\hline Outros & 55 & 34,6 & 104 & 65,4 & 159 & 100,0 & \\
\hline \multicolumn{8}{|l|}{ Renda (em Reais) } \\
\hline$>1.000,00$ & 111 & 36,3 & 195 & 63,7 & 306 & 100,0 & 0,005 \\
\hline $501,00-1.000,00$ & 90 & 50,0 & 90 & 50,0 & 180 & 100,0 & \\
\hline$\leq 500,00$ & 44 & 49,4 & 45 & 50,6 & 89 & 100,0 & \\
\hline \multicolumn{8}{|l|}{ Estado civil } \\
\hline Com companheiro & 171 & 41,0 & 246 & 59,0 & 417 & 100,0 & 0,207 \\
\hline Sem companheiro & 74 & 46,8 & 84 & 53,2 & 158 & 100,0 & \\
\hline \multicolumn{8}{|l|}{ Índice de massa corporal (kg/m²) } \\
\hline $18,5-29,9$ & 206 & 45,8 & 244 & 54,2 & 450 & 100,0 & 0,016 \\
\hline$\geq 30,0$ & 24 & 32,0 & 51 & 68,0 & 75 & 100,0 & \\
\hline$<18,5$ & 5 & 23,8 & 16 & 76,2 & 21 & 100,0 & \\
\hline \multicolumn{8}{|l|}{ Dor impedindo a atividade física } \\
\hline Nenhuma ou muito pouca & 220 & 44,0 & 280 & 56,0 & 500 & 100,0 & 0,082 \\
\hline Dor moderada ou extrema & 25 & 33,3 & 50 & 66,7 & 75 & 100,0 & \\
\hline \multicolumn{8}{|l|}{ Dificuldade de locomoção } \\
\hline Nenhuma & 231 & 44,6 & 287 & 55,4 & 518 & 100,0 & 0,004 \\
\hline Pouca ou muita & 14 & 24,6 & 43 & 75,4 & 57 & 100,0 & \\
\hline \multicolumn{8}{|l|}{ Energia para o dia-a-dia } \\
\hline Alguma ou muita & 241 & 43,4 & 314 & 56,6 & 555 & 100,0 & 0,037 \\
\hline Nenhuma & 4 & 20,0 & 16 & 80,0 & 20 & 100,0 & \\
\hline \multicolumn{8}{|c|}{ Como se sente em relação à saúde } \\
\hline Satisfeito & 238 & 43,5 & 309 & 56,5 & 547 & 100,0 & 0,053 \\
\hline Insatisfeito & 7 & 25,0 & 21 & 75,0 & 28 & 100,0 & \\
\hline
\end{tabular}

* Valor $p$ do $\chi^{2}$ de Yates.

\section{Discussão}

De acordo com a literatura, esta é a primeira pesquisa de base populacional que investigou a prevalência de inatividade física em um município de pequeno porte no Brasil. Joaçaba é uma cidade que apresenta mortalidade infantil igual a 18,1/mil nascidos vivos e índice de desenvolvimento humano (IDH) municipal igual a 0,8612 .

A excelente taxa de resposta obtida no estudo e a seleção da amostra contribuem para a validade interna do estudo, diminuindo a chance de ocorrência de erros sistemáticos. A amostra 
Análise de regressão de Poisson para inatividade física. Joaçaba, Santa Catarina, Brasil; 2003.

\begin{tabular}{|c|c|c|c|c|}
\hline Variáveis (categorias) & $\begin{array}{l}\text { Razão de prevalência } \\
\text { (IC95\%) não ajustada }\end{array}$ & $p$ * & $\begin{array}{l}\text { Razão de prevalência } \\
\text { (IC95\%) ajustada }\end{array}$ & 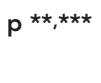 \\
\hline \multicolumn{5}{|l|}{ Idade (anos) } \\
\hline$\leq 29$ & 1,0 & 0,027 & 1,0 & 0,04 \\
\hline $30-59$ & $1,5(1,0-2,2)$ & & $1,2(1,0-1,4)$ & \\
\hline \multicolumn{5}{|l|}{ Escolaridade (anos) } \\
\hline 8 ou mais & 1,0 & 0,003 \# & Excluído & \\
\hline $5-7$ & $0,6(0,4-0,9)$ & & & \\
\hline$<5$ & $0,5(0,3-0,8)$ & & & \\
\hline \multicolumn{5}{|l|}{ Ocupação } \\
\hline Trabalha e/ou aposentado & 1,0 & 0,016 & 1,0 & 0,009 \\
\hline Outros & $1,5(1,0-2,3)$ & & $1,2(1,0-1,4)$ & \\
\hline \multicolumn{5}{|l|}{ Renda (em Reais) } \\
\hline$>1.000,00$ & 1,0 & $0,006 \#$ & 1,0 & 0,001 \\
\hline $501,00-1.000,00$ & $0,6(0,4-0,8)$ & & $0,75(0,6-0,8)$ & \\
\hline$\leq 500,00$ & $0,6(0,4-0,9)$ & & $0,73(0,5-0,9)$ & \\
\hline \multicolumn{5}{|l|}{ Índice de massa corporal (kg/m²) } \\
\hline $18,5-29,9$ & 1,0 & $0,001 \#$ & 1,0 & 0,001 \\
\hline$\geq 30$ & $1,8(1,1-3,0)$ & & $1,2(1,0-1,4)$ & \\
\hline$<18,5$ & $2,7(1,0-7,5)$ & & $1,5(1,2-1,9)$ & \\
\hline \multicolumn{5}{|l|}{ Dor impedindo atividade física } \\
\hline Nenhuma ou muito pouca & 1,0 & 0,083 & Excluído & \\
\hline Dor moderada ou extrema & $1,6(0,9-2,6)$ & & & \\
\hline \multicolumn{5}{|l|}{ Dificuldade de locomoção } \\
\hline Nenhuma & 1,0 & 0,004 & 1,0 & 0,038 \\
\hline Pouca ou muita & $2,4(1,3-4,6)$ & & $1,2(1,0-1,4)$ & \\
\hline \multicolumn{5}{|l|}{ Energia para o dia a dia } \\
\hline Alguma ou muita & 1,0 & 0,047 & Excluído & \\
\hline Nenhuma & $3,1(1,0-9,3)$ & & & \\
\hline \multicolumn{5}{|c|}{ Como se sente em relação à saúde } \\
\hline Satisfeito & 1,0 & 0,060 & Excluído & \\
\hline Insatisfeito & $2,3(1,0-5,5)$ & & & \\
\hline
\end{tabular}

* Valor de p não ajustado ( $p$ do qui-quadrado);

** Valor do p de tendência ajustado;

*** Valor de p controlado por sexo;

\# Valor do p de tendência.

reproduziu a estrutura populacional do município, segundo gênero e faixa etária 7, garantindo a extrapolação dos resultados para a população como um todo. Contudo, deve-se ter o cuidado ao generalizar os resultados desta pesquisa para outras populações, em face das diferenças no padrão cultural e estilo de vida específicos desta população.

O IPAQ é um questionário recentemente validado numa amostra da população brasileira 9 e vem sendo utilizado em alguns estudos no país 9,13,14. Contém perguntas relativas à freqüência e à duração da realização de atividades físicas de caminhada, atividades de intensidade moderada e vigorosa.

A inatividade física encontrada $(57,4 \%)$ foi maior do que as observadas na Suécia (32\%) e na Irlanda (35\%) 15, apesar de as metodologias empregadas nos estudos feitos em ambos os países terem considerado apenas as atividades físicas recreacionais.

Em Pelotas, Rio Grande do Sul, Brasil, verificou-se prevalência de inatividade física de $80,7 \%$ 16. Em Santa Catarina, $68,1 \%$ dos trabalhadores de indústrias eram inativos fisicamente 17 . Deve-se observar que a pesquisa realizada 
em Pelotas considerou as atividades somente no tempo de lazer, em indivíduos de 20 a 69 anos de idade. Os indivíduos que são inativos durante o tempo de lazer podem ser mais ativos em outros contextos. Outros estudos nacionais de base populacional encontraram prevalências semelhantes à da nossa pesquisa 6,18 como, por exemplo, em Florianópolis, onde 46,1\% dos indivíduos de 25 a 49 anos foram considerados insuficientemente ativos 19 .

Em Mafra, Santa Catarina, observou-se que $89,9 \%$ dos adultos eram suficientemente ativos 20. Contudo, os autores utilizaram o IPAQ em sua versão longa. A versão curta desse questionário parece superestimar a inatividade física quando se compara com a sua versão longa 18 .

Ao contrário do que foi observado em outros estudos $16,20,21,22,23$, verificou-se a inatividade física associada a indivíduos com renda maior. Hallal et al. 18 também evidenciaram maior atividade física em indivíduos pertencentes a níveis sócio-econômicos mais baixos.

A inatividade física apresentou-se associada aos indivíduos sem trabalho e/ou sem aposentadoria. Na Alemanha, as pessoas empregadas apresentaram uma freqüência maior de atividade física durante o lazer 23. Provavelmente, aqueles com rendas mais altas apresentam maior possibilidade de participar de programas de promoção da saúde, com comportamentos mais ativos.

Apesar de outros estudos terem mostrado diferenças entre sexo e nível de atividade física $16,20,21$, esta diferença não foi observada na nossa pesquisa, assim como no estudo de Hallal et al. 18 realizado em adultos de Pelotas.

Segundo Kemper 24, os níveis de atividade física diminuem na vida adulta, condição semelhante observada na presente pesquisa em outros estudos 6,16,18,20,21,23.
Nosso resultado confirma o de outros estudos 18 quanto à relação entre IMC e inatividade física. Porém, estudos com características transversais não permitem verificar a relação de temporalidade entre duas variáveis, devendo, portanto, ser considerada a possibilidade de causalidade reversa entre inatividade física e IMC, o mesmo ocorrendo com relação à capacidade de locomoção.

Inatividade física e o estado civil das pessoas não se apresentaram associados, corroborando outro estudo brasileiro 16. Entretanto, Barros \& Nahas 17 verificaram que os indivíduos solteiros apresentaram-se menos inativos durante o lazer quando comparados com indivíduos casados.

Percepção negativa do seu estado de saúde apresentou-se associada à inatividade física, reforçando a idéia de que a atividade física tem impacto positivo sobre a percepção da saúde dos indivíduos 17. No nosso estudo, não foi possível identificar qual o tipo de atividade física esteve relacionada à percepção da saúde.

No Município de Joaçaba, as condições demográficas e ambientais, entre elas, a presença de muitos morros e o tipo de ocupação, como a construção civil e metal-mecânica, podem ter influenciado os resultados. Também deve ser considerado o bom suporte social dos indivíduos, as características da cidade de colonização italiana e alemã e a forma de deslocamento das pessoas, muitas vezes sem a utilização de algum meio de transporte.

Conhecer o perfil da atividade física nos diferentes contextos da vida dos indivíduos tem uma implicação importante na definição de políticas públicas de saúde do município podendo, portanto, ser cuidadosamente considerado em futuros estudos. 


\section{Resumo}

Estimou-se a prevalência de inatividade física na população adulta do Município de Joaçaba, Santa Catarina, Brasil, e os fatores associados. Foi realizado um estudo transversal de base populacional domiciliar, com indivíduos de 20 a 59 anos, de ambos os sexos ( $n=579)$. Utilizou-se o Questionário Internacional de Atividade Física (IPAQ), na forma curta, para estimar o nível de atividade física. Foram realizadas análises descritivas e regressão de Poisson. A taxa de resposta foi de 99,6\%. A prevalência de indivíduos classificados como inativos fisicamente foi de 57,4\% (IC95\%: 53,461,4). A presença de inatividade física apresentou-se associada positivamente a indivíduos que não trabalhavam ou não eram aposentados, àqueles com renda familiar superior a mil reais mensais, idade superior a trinta anos, presença de índice de massa corporal maior ou menor que o padrão normal e a indivíduos que auto-avaliaram dificuldades de locomoção, independentemente do sexo. A prevalência de inatividade física encontrada foi menor que nos outros estudos de base populacional já realizados no país. Os resultados podem auxiliar a implantação de políticas públicas que estimulem a população à prática regular de exercícios.

Atividade Motora; Adulto; Prevalência

\section{Referências}

1. Baptista PB. Epidemiologia da atividade física. Rev SOCERJ 2000; 8:173-4.

2. Ministério da Saúde. Programa Nacional de Promoção da Atividade Física "Agita Brasil": atividade física e sua contribuição para a qualidade de vida. Rev Saúde Pública 2002; 36:254-6.

3. American Heart Association. Statement on exercise. Circulation 1992; 86:2726-30.

4. Ministério da Saúde. Agita Brasil: guia para agentes multiplicadores. Brasília: Ministério da Saúde; 2002.

5. Monteiro CA, Conde WL, Matsudo SM, Matsudo VR, Bonseñor IM, Lotufo PA. A descriptive epidemiology of leisure-time physical activity in Brazil, 1996-1997. Rev Panam Salud Pública 2003; 14:24654.

6. Matsudo SM, Matsudo VR, Araújo T, Andrade D, Andrade E, Oliveira LC, et al. Nível de atividade física da população do Estado de São Paulo: análise de acordo com o gênero, idade, nível sócio-econômico, distribuição geográfica e de conhecimento. Revista Brasileira de Ciência e Movimento 2002; 10:41-50.

\section{Colaboradores}

E. Baretta participou da revisão da literatura, elaboração do projeto, desenvolvimento da proposta de trabalho, trabalho de campo e redação do artigo. K. G. Peres analisou os dados, contribuiu com a orientação do projeto e revisão final do artigo. M. Baretta contribuiu na elaboração dos instrumentos, do trabalho de campo e na discussão do artigo.
7. Instituto Brasileiro de Geografia e Estatística. Censo demográfico 2000. Rio de Janeiro: Instituto Brasileiro de Geografia e Estatística; 2000.

8. Heyward VH, Stolarczyk LM. Avaliação da composição corporal. São Paulo: Editora Manole; 2000.

9. Matsudo SM, Araújo T, Matsudo VR, Andrade D, Andrade E, Oliveira LC, et al. Questionário Internacional de Atividade Física (IPAQ): estudo de validade e reprodutibilidade no Brasil. Rev Bras Ativ Fís Saúde 2001; 6:5-18.

10. International Physical Activity Questionnaire. Downloadable questionnaires. http://www.ipaq. ki.se/IPAQ.asp?mnu_sel=DDE\&pg_sel=DDE (acessado em Mar/2003).

11. Barros AJ, Hirakata VN. Alternatives for logistic regression in cross-sectional studies: an empirical comparison of models that directly estimate the prevalence ratio. BMC Med Res Methodol 2003; $3: 21$. 
12. Instituto Brasileiro de Geografia e Estatística/ Programa das Nações Unidas para o Desenvolvimento/Instituto de Pesquisa Econômica Aplicada/ Fundação João Pinheiro. Atlas do desenvolvimento humano no Brasil [CD-ROM]. Brasília: Instituto Brasileiro de Geografia e Estatística/Programa das Nações Unidas para o Desenvolvimento/Instituto de Pesquisa Econômica Aplicada/Fundação João Pinheiro; 2000.

13. Pardini R, Matsudo S, Araújo T, Matsudo V, Andrade E, Braggion G et al. Validação do Questionário Internacional de Nível de Atividade Física (IPAQ - versão 6): estudo piloto em adultos jovens brasileiros. Revista Brasileira de Ciência e Movimento 2001; 9:45-51.

14. Hallal PC, Matsudo SM, Matsudo VKR, Araújo TL, Andrade DR, Bertoldi AD. Physical activity in adults from two Brazilian areas: similarities and differences. Cad Saúde Pública 2005; 21:573-80.

15. Vouri IM. Health benefits of physical activity with special reference to interaction with diet. Public Health Nutr 2001; 4:517-28.

16. Dias-da-Costa JS, Hallal PC, Wells JCK, Daltoé T, Fuchs SC, Menezes AMB, et al. Epidemiology of leisure-time physical activity: a population-based study in southern Brazil. Cad Saúde Pública 2005; 21:275-82.

17. Barros MVG, Nahas MV. Comportamentos de risco, auto-avaliação do nível de saúde e percepção de estresse entre trabalhadores da indústria. Rev Saúde Pública 2001; 35:554-63.
18. Hallal PC, Victora CG, Wells JCK, Lima RC. Physical inactivity: prevalence and associated variables in Brazilian adults. Med Sci Sports Exerc 2003; 38:1894-900.

19. Instituto Nacional de Câncer. Inquérito domiciliar sobre comportamentos de risco e morbidade referida de doenças e agravos não transmissíveis: Brasil, 15 capitais e Distrito Federal, 2002-2003. Rio de Janeiro: Instituto Nacional de Câncer; 2004.

20. Flores A. Prevalência da inatividade física e outros fatores de risco relacionados à saúde na população adulta urbana de Mafra - SC [Dissertação de Mestrado]. Florianópolis: Centro de Desportos, Universidade Federal de Santa Catarina; 2002.

21. Bernstein MS, Costanza MC, Morabia A. Phisical activity of urban adults: a general population survey in Geneva. Soz Praventivmed 2001; 46:49-59.

22. Domingues MR, Araújo CLP, Gigante DP. Conhecimento e percepção sobre exercício físico em uma população adulta urbana do sul do Brasil. Cad Saúde Pública 2004; 20:204-15.

23. Mensink GBM, Loose N, Oomen C. Physical activity and its association with other lifestyle factors. Eur J Epidemiol 1997; 13:771-8.

24. Kemper H. The natural history of physical activity and aerobic fitness in teenagers. In: Dishman $\mathrm{R}$, editor. Advances in exercise adherence. Champaign: Human Kinetics; 1994. p. 293-318.

Recebido em 31/Mar/2006

Versão final reapresentada em 14/Set/2006 Aprovado em 10/Jan/2007 